\title{
Take Taobao as an Example to Discuss the Comprehensive Evaluation Methods for C2C E-Commerce Websites' Competitiveness
}

\author{
Ming Li \\ JiLin Business And Technology College, ChangChun, China \\ 120857715@qq.com
}

Keywords: Taobao, Competitiveness, Evaluation Research

\begin{abstract}
C2C e-commerce has been in the rapid development in recent years, and the representative websites in China include Taobao (www.taobao.com), eBay (www.e-bay.com), Jinshan Amazon (www.amazon.com.cn) and so on. The investigation from Internet Research and Development Center of CASS shows that C2C has become the most striking area for the competition of e-commerce, and the proportion of Internet users shopping online in China has exceeded the average level of the Asia-Pacific region. However, during the rapid development, the competition in the field of $\mathrm{C} 2 \mathrm{C}$ e-commerce is becoming increasingly fierce. For the C2C e-commerce websites, fully evaluating and understanding its own competitiveness are the premises for enterprises to enhance the competitiveness and gain competitive advantages, and now it is also a problem crying out for solutions. Through the analysis of Taobao, the paper verifies the feasibility of proposed comprehensive evaluation index system and evaluation methods for C2C e-commerce websites.
\end{abstract}

\section{Introduction}

The e-commerce website evaluation is one of the hot fields of electronic commerce. The existing work has been focused on the evaluation for e-commerce websites, such as B2B[1], B2C and B2G. Researchers have proposed a variety of evaluation methods which have certain significance for competitiveness evaluation of C2C e-commerce websites. From the point of view of acquisitive methods for information and data required by evaluation, the existing methods can be classified into three categories. The method relying on subjective evaluation refers to evaluating the merit and defect of a website by a subjective judgment. The expert evaluation and questionnaires are two ways commonly used in this method. The expert evaluation method which can give a comprehensive evaluation to each selected website has an advantage of pooling collective wisdom, but whether it is an online survey or an expert evaluation, subjective factors always have a significant impact during the evaluation process. Therefore, the subjective evaluation method is suitable as a method for public to understanding C2C e-commerce websites or for C2C e-commerce websites to expand their influence. The objective evaluation method refers to evaluating the merit and defect of a website by considering the actual operation of the website. The most commonly used method is the statistic of website flow indicators. But overall, the statistic of website flow indicators is too simple for the competitiveness evaluation of $\mathrm{C} 2 \mathrm{C}$ e-commerce websites, and the flow rank can't fully reflect the competitiveness of C2C e-commerce websites. The comprehensive evaluation method refers to a method which first establishes a comprehensive evaluation system and then collects data by various methods, such as technical measurements, expert surveys and user surveys, and at last exploits and analyzes the data and related information by qualitative and quantitative methods, such as a comparative analysis and a model analysis. The comprehensive evaluation method is a relatively scientific evaluation method. According to our findings, researches used a comprehensive evaluation method to evaluate the core competitiveness of C2C e-commerce websites are still rare, it is also rarely reported especially in the aspect of evaluation models for competitiveness of $\mathrm{C} 2 \mathrm{C}$ e-commerce websites. According to the existing information, the evaluation of most $\mathrm{C} 2 \mathrm{C}$ e-commerce websites is only in the stage of market research and data collection. A few of literature analyzed the system of evaluation indicators of C2C e-commerce 
websites and evaluated the credit problems of C2C e-commerce websites, but there is little study reported to systematically evaluate $\mathrm{C} 2 \mathrm{C}$ e-commerce websites from a competitive point of view.

Currently, Taobao, accounting for more than $70 \%$ domestic market share, is well-deserved the first C2C e-commerce website in China. This paper takes Taobao (www.taobao.com) as an example to verify the feasibility of the comprehensive evaluation method of C2C e-commerce websites' competitiveness.

\section{The paper comprehensively evaluated the competitiveness of $\mathrm{C} 2 \mathrm{C}$ e-commerce websites by the method of the Analytic Hierarchy Process (AHP) and the method of the Multistage Fuzzy Comprehensive Evaluation.}

The method of the AHP and the method of the Multistage Fuzzy Comprehensive Evaluation are two common evaluation methods[2]. Both methods have their advantages and disadvantages. The method of the AHP which can avoid arbitrariness and subjectivity brought from scoring ways of experts is suitable for the consideration of constructing evaluation indicators, while the method of the Multistage Fuzzy Comprehensive Evaluation is a comprehensive multi-dimensional evaluation method which can give the final result by multi-dimensional methods, avoiding inaccuracies brought from a traditional binary mode (good/bad) or a single value calculation (how many points)[3]. Because in the actual evaluation, many examples of evaluation are often difficult to simply indicate whether it is good or bad by a single score, to determine the effectiveness ultimately usually requires to integrating indicators from many aspects and combining the characteristics of fields.

The final evaluation result obtained by the method of the Multistage Fuzzy Comprehensive Evaluation is an alternative set (very good, good, medium, poor, very poor). According to the finally generated alternative set, we give two explanations to this method: one explanation method is to directly weight sum of the results of the alternative set and present a normalization to deal with the results of the alternative set, for example, in the achieved method, if the value gained from the process of weight sum and normalization is above 80, the evaluation result is "good"; another method is to directly interpret the alternative set into a set of assessment opinions from different experts.

First, the evaluation grade is divided into five categories: corresponded to very good, good, medium, poor, and very poor, respectively. We randomly selected 50 Taobao users to vote for the competitiveness of Taobao, the vote is shown in Table 1.

Table 1 The Fuzzy Relation Matrix for Taobao’s Competitiveness

\begin{tabular}{l|l|l|l|l|l|l}
\hline \multicolumn{2}{c}{ Factors } & $\begin{array}{l}\text { Very } \\
\text { Good }\end{array}$ & Good & Medium & Poor & $\begin{array}{l}\text { Very } \\
\text { Poor }\end{array}$ \\
\hline \multirow{4}{*}{ Safety } & Privacy & 11 & 19 & 12 & 8 & 0 \\
\cline { 2 - 7 } & Network Security & 13 & 21 & 9 & 7 & 0 \\
\cline { 2 - 7 } & Network Authentication & 20 & 16 & 9 & 5 & 0 \\
\hline \multirow{5}{*}{ Services } & Communication & 32 & 19 & 9 & 0 & 0 \\
\cline { 2 - 7 } & Payment Platform & 21 & 19 & 8 & 2 & 0 \\
\cline { 2 - 7 } & Logistics System & 7 & 13 & 19 & 11 & 0 \\
\cline { 2 - 7 } & Return and Change Service & 9 & 30 & 10 & 1 & 0 \\
\cline { 2 - 7 } & Credit Evaluation System & 6 & 16 & 21 & 7 & 0 \\
\hline \multirow{5}{*}{ Information } & Applicability & 7 & 12 & 23 & 8 & 0 \\
\hline
\end{tabular}




\begin{tabular}{c|l|l|l|l|l|l}
\hline \multirow{5}{*}{ Technologies } & Comprehensiveness & 14 & 21 & 11 & 4 & 0 \\
\cline { 2 - 7 } & Accuracy & 24 & 12 & 10 & 4 & 0 \\
\cline { 2 - 7 } & Novelty & 21 & 15 & 12 & 2 & 0 \\
\cline { 2 - 7 } & Site Response Speed & 9 & 16 & 20 & 5 & 0 \\
\cline { 2 - 7 } & User Interface & 27 & 18 & 5 & 0 & 0 \\
\cline { 2 - 7 } & Search Approaches & 6 & 21 & 18 & 5 & 0 \\
\cline { 2 - 7 } & Search Results & 8 & 12 & 22 & 8 & 0 \\
\cline { 2 - 7 } & Network Visibility & 25 & 17 & 6 & 2 & 0 \\
\cline { 2 - 7 } & Network Influence & 30 & 16 & 4 & 0 & 0 \\
\cline { 2 - 7 } & Market Share & 39 & 11 & 0 & 0 & 0 \\
\hline
\end{tabular}

The numbers in Table 3 indicate the number of voters. According to Table 3, we can get the membership degree of each factor on various levels, and then calculate the fuzzy relation matrix, and finally get a comprehensive evaluation result. The secondary index calculation of Safety (A1), for example, we can know the relative weight value of B1, B2 and B3 is $(0.35,0.35$ and 0.3$)$ respectively according to Table 2, so we can get an alternative set of Safety (A1)[4]:

$$
\begin{aligned}
& (\mathrm{r} 11, \mathrm{r} 12, \mathrm{r} 13, \mathrm{r} 14, \mathrm{r} 15)=(0.35,0.35,0.3) \\
& \left(\begin{array}{lllll}
11 / 50 & 19 / 50 & 12 / 50 & 8 / 50 & 0 \\
13 / 50 & 21 / 50 & 9 / 50 & 7 / 5 & 0 \\
20 / 50 & 16 / 50 & 9 / 50 & 5 / 50 & 0
\end{array}\right)
\end{aligned}
$$

Similarly: We can calculate the alternative sets for the remaining four primary indexes, and then get a fuzzy relation matrix $\mathrm{R}$ :

$$
R=\left(\begin{array}{ccccc}
0.288 & 0.376 & 0.201 & 0.135 & 0 \\
0.256 & 0.294 & 0.278 & 0.092 & 0 \\
0.323 & 0.291 & 0.292 & 0.094 & 0 \\
0.273 & 0.323 & 0.316 & 0.088 & 0 \\
0.670 & 0.274 & 0.048 & 0.008 & 0
\end{array}\right)
$$

According to the relative weight vectors of A1, A2, A3, A4 and A5 from Table 2, we can calculate the final fuzzy comprehensive evaluation result:

$$
\begin{aligned}
& \mathrm{B}=\mathrm{A} \# \mathrm{R}=\left(\begin{array}{lllll}
0.42 & 0.26 & 0.1 & 0.16 & 0.06
\end{array}\right) \\
& \left(\begin{array}{ccccc}
0.288 & 0.376 & 0.201 & 0.135 & 0 \\
0.256 & 0.294 & 0.278 & 0.092 & 0 \\
0.323 & 0.291 & 0.292 & 0.094 & 0 \\
0.273 & 0.323 & 0.316 & 0.088 & 0 \\
0.67 & 0.274 & 0.048 & 0.008 & 0
\end{array}\right)
\end{aligned}
$$

In this paper, the evaluation result can be interpreted as: a. $30.4 \%$ of experts believe that the competitiveness of Taobao is "very good"; b. 33.2\% of experts believe that the competitiveness of Taobao is "better"; c. $23.9 \%$ of experts believe that the competitiveness of Taobao is "medium"; $d$. 
$10.5 \%$ of experts believe that the competitiveness of Taobao is "poor"; e. no experts believe that the competitiveness of Taobao is "very poor".

\section{Conclusion}

The evaluation index system for C2C e-commerce websites' competitiveness, especially the evaluation methods, is the focal problem of the competitiveness evaluation of C2C e-commerce websites. Based on the analysis of C2C e-commerce websites' competitiveness, this paper provides a layer evaluation index system of competitiveness for $\mathrm{C} 2 \mathrm{C}$ e-commerce websites and an evaluated method of competitiveness for $\mathrm{C} 2 \mathrm{C}$ e-commerce websites based on the method of the AHP and the method of the Multistage Fuzzy Comprehensive Evaluation. The theoretical deduction proved that this method is suitable for competitiveness evaluation of C2C e-commerce websites. Based on this[5], we have developed a competitiveness evaluation tool for C2C e-commerce websites, achieving a computer automation of competitiveness evaluation work of $\mathrm{C} 2 \mathrm{C}$ e-commerce websites. Further work will be focused on the determination of the membership degree of each underlying index. The membership degree of the underlying index is a key factor which can influence the result of the Multistage Fuzzy Comprehensive Evaluation method, but because the membership degree of different indexes are very different (eg, some is believed that bigger is better, while some is believed that smaller is better), we need to study different determination methods for different types of indexes.

\section{References}

[1] J.X. Yul. Chinese E-Commerce (2004-2005). Beijing: Economic Science Press, 2006

[2] X.Y. Zhang, L. Wang and P. Zhang, et al. Problems and Solutions in the Development of China’s C2C E-Commerce. Intelligence Magazine, 2005 (6): 78-80

[3] J.M. Xu. Intellectual Capitan and the Core Competitiveness of E-Commerce Enterprises. Modern Information: 2007 (10): 196-198

[4] A K El-Aleem,W F El-Wahed, et al. Efficiency Evaluation of E-Commerce Websites. Proc. Of WEC.05: 2005:20 -23

[5] M.Z. Yang, Y.L. Zhuang. Study on Evaluation of Enterprise E-Commerce Websites. Intelligence Magazine, 2009 (2): 111-114. 